\title{
Lipid-lowering agents in chronic kidney disease: do fibrates have a role?
}

Yuli Huang and Yunzhao Hu

We read the Review by Kahn et al. (Management of cardiovascular disease in patients with kidney disease. Nat. Rev. Cardiol. 10, 261-273; 2013) $)^{1}$ with great interest. We wish to discuss the role of fibrates in the management of dyslipidaemia in patients with chronic kidney disease (CKD).

Statins substantially decrease cardiovascular disease $(\mathrm{CVD})$ and mortality in patients with CKD who do not require dialysis. ${ }^{2-4}$ However, because serum levels of triglyceride-rich lipoproteins are increased in patients with CKD, particularly in those with diabetic nephropathy, non-HDL cholesterol could be a better biomarker of dyslipidaemia than LDL cholesterol in this population. ${ }^{5}$

The amount by which triglyceride is lowered is approximately doubled if fibrates are used in addition to statins. Thus, fibrates are a second-line preventive therapy for patients in the general population with hypertriglyceridaemia and a residual elevation in the level of non-HDL cholesterol. However, their role in patients with CKD has yet to be defined. ${ }^{6}$ Investigators in the Fenofibrate Intervention and Event Lowering in Diabetes (FIELD) study ${ }^{7}$ randomly allocated 9,795 participants with type 2 diabetes mellitus to either fenofibrate ( $200 \mathrm{mg}$ daily) or placebo, with a median follow-up period of 5 years. In this study, fenofibrate treatment reduced total cardiovascular events (HR 0.89, 95\% CI $0.80-0.99) .^{7}$ This benefit did not differ significantly between groups with different estimated glomerular filtration rates. The investigators concluded that fenofibrate should be considered as an additional therapeutic option, along with conventional risk-factor management, to further reduce CVD events and mortality and provide renoprotection to patients with diabetes, as well as to those with moderate renal impairment. ${ }^{8}$ By contrast, in the Action to Control Cardiovascular Risk in Diabetes
(ACCORD) lipid trial, ${ }^{9}$ the addition of fenofibrate ( $160 \mathrm{mg}$ daily) to simvastatin treatment (10-40 $\mathrm{mg}$ daily) was assessed in 5,518 participants with diabetes. No significant difference was found in the primary outcome of nonfatal myocardial infarction, nonfatal stroke, or cardiovascular death. In a meta-analysis, fibrates reduced the risk of albuminuria progression in patients with diabetes, and reduced the risk of major cardiovascular events and cardiovascular death (but not all-cause mortality) in patients with an estimated glomerular filtration rate of $30-59.9 \mathrm{ml} / \mathrm{min} / 1.73 \mathrm{~m}^{2} .^{10}$

Fibrates also increase the serum creatinine level after therapy initiation, but this effect could be a response to the blunting of creatinine secretion rather than true renal injury. ${ }^{11}$ In post-trial analysis of the FIELD ${ }^{12}$ and $\mathrm{ACCORD}^{9}$ data sets, acute changes in the creatinine level with fibrate therapy were not associated with adverse effects on major clinical renal outcomes, and indeed suggest the possibility of long-term renal benefit. ${ }^{10}$

Taken together, fibrates are likely to improve lipid profiles and prevent CVD in patients with CKD. However, the available data come from subgroup analyses of trials in broad populations, and no data are currently available for individuals with end-stage renal disease. Therefore, large-scale, prospective, randomized trials to assess the role of fibrates in reducing cardiovascular risk in patients with CKD, especially those with diabetic nephropathy, are urgently needed.

Department of Cardiology, the Affiliated Hospital at Shunde (the First People's Hospital of Shunde), Southern Medical University, Penglai Road 1, Daliang Town, Shunde District, Foshan 528300, China (Y. Huang, Y. Hu). Correspondence to: $Y$. Hu huyunzha04406@163.com

\section{Acknowledgements}

The authors of this manuscript are funded by the Medical Scientific Research Grant of the Health
Ministry of Guangdong province, China (No. B2011310, No. A2012663), and the Scientific Research Fund of Foshan, Guangdong, China (No. 201208210).

Competing interests

The authors declare no competing interests

1. Kahn, M. R., Robbins, M. J., Kim, M. C. \& Fuster, V. Management of cardiovascular disease in patients with kidney disease. Nat. Rev. Cardiol. 10, 261-273 (2013).

2. Palmer, S. C. et al. Benefits and harms of statin therapy for persons with chronic kidney disease: a systematic review and meta-analysis. Ann. Intern. Med. 157, 263-275 (2012).

3. Upadhyay, A. et al. Lipid-lowering therapy in persons with chronic kidney disease: a systematic review and meta-analysis. Ann. Intern. Med. 157, 251-262 (2012).

4. Barylski, M. et al. Statins decrease all-cause mortality only in CKD patients not requiring dialysis therapy-a meta-analysis of 11 randomized controlled trials involving 21,295 participants. Pharmacol. Res. 72, 35-44 (2013).

5. Nitta, K. Clinical assessment and management of dyslipidemia in patients with chronic kidney disease. Clin. Exp. Nephrol. 16, 522-529 (2012).

6. McCullough, P. A. \& Di Loreto, M. J. Fibrates and cardiorenal outcomes. J. Am. Coll. Cardiol. 60, 2072-2073 (2012).

7. Keech, A. et al. Effects of long-term fenofibrate therapy on cardiovascular events in 9795 people with type 2 diabetes mellitus (the FIELD study): randomised controlled trial. Lancet $\mathbf{3 6 6}$, 1849-1861 (2005).

8. Ting, R. D. et al. Benefits and safety of longterm fenofibrate therapy in people with type 2 diabetes and renal impairment: the FIELD study. Diabetes Care 35, 218-225 (2012).

9. Mychaleckyj, J. C. et al. Reversibility of fenofibrate therapy-induced renal function impairment in ACCORD type 2 diabetic participants. Diabetes Care 35, 1008-1014 (2012).

10. Jun, M. et al. Effects of fibrates in kidney disease: a systematic review and metaanalysis. J. Am. Coll. Cardiol. 60, 2061-2071 (2012).

11. Udani, S. M. \& Bakris, G. L. Do fibrates truly preserve kidney function? Nat. Rev. Endocrinol. 7, 130-131 (2011).

12. Davis, T. M. et al. Effects of fenofibrate on renal function in patients with type 2 diabetes mellitus: the Fenofibrate Intervention and Event Lowering in Diabetes (FIELD) study. Diabetologia 54, 280-290 (2011). 